\title{
Domain-wide regulation of DNA replication timing during mammalian development
}

\author{
Benjamin D. Pope • Ichiro Hiratani • \\ David M. Gilbert
}

Published online: 15 December 2009

(C) Springer Science+Business Media B.V. 2009

\begin{abstract}
Studies of replication timing provide a handle into previously impenetrable higher-order levels of chromosome organization and their plasticity during development. Although mechanisms regulating replication timing are not clear, novel genome-wide studies provide a thorough survey of the extent to which replication timing is regulated during most of the early cell fate transitions in mammals, revealing coordinated changes of a defined set of $400-800 \mathrm{~kb}$ chromosomal segments that involve at least half the genome. Furthermore, changes in replication time are linked to changes in sub-nuclear organization and domain-wide transcriptional potential, and tissue-specific replication timing profiles are conserved from mouse to human, suggesting that the program has developmental significance. Hence, these studies have provided a solid foundation for linking megabase level chromosome structure to function, and suggest a central role for replication in domain-level genome organization.
\end{abstract}

Keywords Replication Timing $\cdot$ Mammalian Development $\cdot$ Cell Differentiation $\cdot$ Chromosome Domains $\cdot$ Nuclear Organization $\cdot$ Chromatin Structure $\cdot$ Sub-nuclear Positioning $\cdot$ Transcriptional Competence

Responsible Editors: Marie-Noëlle Prioleau and Dean Jackson.

B. D. Pope $\cdot$ I. Hiratani $\cdot$ D. M. Gilbert $(\bowtie)$

Department of Biological Science, Florida State University, Tallahassee, FL 32306, USA

e-mail: gilbert@bio.fsu.edu

\begin{abstract}
Abbreviations
$\mathrm{R} / \mathrm{G} \quad$ reverse/Giemsa

ESC embryonic stem cell

$\mathrm{Xi}$ inactive $\mathrm{X}$ chromosome

iPSC induced pluripotent stem cell

TDP timing decision point

ODP origin decision point

HP1 heterochromatin protein 1

HMG I/Y high mobility group proteins I and Y

LCR locus control region

LINE-1 long interspersed nuclear element 1
\end{abstract}

\section{Introduction}

During development, cell division and differentiation ultimately convert a single totipotent cell into an organized conglomeration of tissues with various specialized functions and features. Along the way, cells are induced to progress down particular paths of differentiation and undergo changes that decrease their responsiveness, or competence, to cues from other cell types (Grimm and Gurdon 2002; Steinbach et al. 1997; Waddington 1940). Though for many years this process was seen as unidirectional and irreversible, the idea that totipotent differentiation potential is completely lost during development was challenged by amphibian cloning studies in the $1960 \mathrm{~s}$ (Gurdon 2006) and later put to rest by the cloning of Dolly (Campbell et al. 1996). Recent work by 
Yamanaka has demonstrated that differentiated cells can regain the potential to respond to cues and change cell type after treatment with defined transcription factors (Takahashi and Yamanaka 2006). Nonetheless, this process is still quite inefficient and questions remain regarding the global mechanisms at work during development to direct cell fate, as well as those acting in the reprogramming process to reverse it (Hochedlinger and Plath 2009).

Accompanying differentiation are specific changes in nuclear genome organization including chromatin structure, chromatin positioning in the nucleus, and the order in which different chromosomal regions are replicated during S-phase known as the replication timing program (Hiratani et al. 2008; Meshorer and Misteli 2006). Though research in recent years has brought to light many players involved in establishing and modifying chromatin organization (Li et al. 2007), no overarching mechanisms have been identified that can explain the connections between these molecular mechanisms and the seemingly concomitant higher-order reorganization events. Nevertheless, changes in higher-order organization correlate with changes in gene expression during development, suggesting that as yet undefined mechanisms regulating higher-order chromosomal structure may contribute to the differentiation process (Fraser and Bickmore 2007). Analysis of replication timing offers a unique approach to view the nuclear programming process during development because it provides a distinct functional property of chromosomes that is clearly regulated at the level of megabase-sized domains. Moreover, while regulatory mechanisms of replication timing remain elusive, evidence points to an intimate relationship between replication timing and chromatin packaging in the nucleus. Here, we review studies that underscore the importance of replication timing's connection to changes in the nucleus that occur during development.

\section{Replication timing is connected to functional and spatial organization of chromatin}

Recent genome-wide analyses of replication timing have confirmed that large blocks of chromatin replicate together (Desprat et al. 2009; Farkash-Amar et al. 2008; Hiratani et al. 2008; Karnani et al. 2007; MacAlpine et al. 2004; Schubeler et al. 2002; White et al. 2004; Woodfine et al. 2004), which we refer to as replication domains. These domains are replicated by synchronously firing origins in close enough proximity to each other to ensure that the length of each domain is replicated within a relatively short period of time (approximately $1 \mathrm{~h}$ in mammals; Gilbert and Gasser 2006). A comparison of these domains with Giemsastained chromomeric bands, which roughly demarcate regions of euchromatin and heterochromatin along the length of a chromosome, reveals general overlap of light-staining $\mathrm{R}$ bands with early-replicating domains and dark-staining $G$ bands with late-replicating domains (Bickmore and Craig 1997; Craig and Bickmore 1993; Holmquist et al. 1982; Latt 1977). Accordingly, chromatin modifications characteristic of euchromatin such as histone acetylation overlap with early-replicating domains (Hiratani et al. 2009). Replication timing is also associated with three-dimensional genome organization in the nucleus. Spatial patterns of DNA replication in the nucleus change dramatically as cells move through S-phase, with early-replicating domains localized in the interior of the nucleus, whereas late-replicating domains are localized to the heterochromatin-rich nuclear and nucleolar peripheries as well as a few central heterochromatic blocks (Berezney et al. 2000). Importantly, this demonstrates a global coupling of sub-nuclear positioning and replication timing of different chromatin domains.

Expectedly, since chromatin structure is associated with transcriptional regulation, correlations between replication timing and gene expression have also been observed. Late-replicating domains packaged in heterochromatin and localized to the nuclear periphery tend to contain fewer genes than early-replicating domains and a much lower percentage of the genes within latereplicating domains are expressed relative to earlyreplicating regions (Hiratani et al. 2008). It is important to note that transcription correlates considerably better with replication timing over large chromosomal domains than at the level of individual genes (MacAlpine and Bell 2005; MacAlpine et al. 2004), suggesting greater transcriptional competence in early versus late-replicating domains. In fact, a recent study integrating the same reporter gene into many different chromosomal sites revealed a domain-wide regulatory mechanism influencing transcriptional output (Gierman et al. 2007). This study demonstrated that domains with high gene density and high GC content, which are features of early-replicating domains, showed higher reporter gene transcription. Naturally, 
however, genes will not be highly transcribed in the absence of gene-specific transcription factors regardless of domain structure, so a domain-wide regulatory mechanism would not be sufficient for transcription. Moreover, when considering regulation at the domain level, one should not be daunted by exceptional genes that appear to break general rules; additional mechanisms exist that locally fine-tune transcription, for example to overcome the condensed nature of a heterochromatic domain [(Hiratani et al. 2008) and references therein]. Collectively, these observations suggest that replication timing is fundamentally associated with establishment of the chromatin structure of broad regions, possibly affecting their accessibility to transcription machineries.

\section{$\mathrm{X}$ chromosome inactivation as a paradigm for developmental regulation}

The strongest evidence for developmental regulation of replication timing and its relationship to transcriptional regulation is the paradigm of $\mathrm{X}$ chromosome inactivation. In female mammalian somatic cells, the dosage-compensated, transcriptionally inactive $\mathrm{X}$ chromosome (Xi) is replicated late in S-phase, distinctly later than when the autosomes and its active X counterpart are replicated (Morishima et al. 1962). While some epigenetic events that accompany Xinactivation are species specific (Chow and Heard 2009), late replication is one of the most conserved features and has served as a cytological X-inactivation index for decades. During random $\mathrm{X}$-inactivation in the embryo proper, the $\mathrm{Xi}$ undergoes a switch to late replication in the postimplantation epiblast, primarily during E5.8-6.3 (Takagi et al. 1982), meaning that the switch to late replication occurs prior to germ layer specification. Importantly, this narrow time window implies that a replication timing switch of the Xi may occur within a single cell cycle (Snow 1977). Accompanying late replication, the $\mathrm{Xi}$ becomes condensed and repositioned toward the nuclear periphery, forming a Barr body (Barr and Bertram 1949). This late-replicating condensed structure is subsequently maintained throughout development, underscoring the stability of this regulation (Chadwick and Willard 2003).

Embryonic stem cell (ESC) differentiation has provided a convenient in vitro model for studying $\mathrm{X}$ - inactivation. Conventional cytogenetic and molecular analyses have revealed that during female ESC differentiation, a switch to late replication occurs within 1-2 days after Xist RNA coating and the appearance of tri-methylation of histone $\mathrm{H} 3$ lysine 27 (H3K27me3) and di-methylation of H3K9 (H3K9me2) on the Xi (Chow and Heard 2009). A chromosomewide histone H4 hypoacetylation and DNA methylation follows the replication timing switch [reviewed in Chow and Heard (2009)]. Analysis of individual genes suggested that transcriptional inactivation initiates immediately following Xist coating. However, recent chromosome-wide analyses reveal a more complex scenario. First, transcriptional silencing takes place over a span of 2-3 weeks and shows promoterdependent regulation (Chow and Heard 2009; Lin et al. 2007). Secondly, cytogenetic studies of the $\mathrm{Xi}$ suggest the presence of two distinct types of repressed chromatin, one enriched for H3K27me3 and the other for $\mathrm{H} 3 \mathrm{~K} 9 \mathrm{me} 3$ almost in a mutually exclusive manner (Chadwick and Willard 2004), suggesting that different domains undergo distinct epigenetic changes. It is not yet known how these domains relate to the replication timing changes along the length of the X-chromosome. Furthermore, the types and distribution of chromatin marks on the $\mathrm{Xi}$ are specific to certain species (Chow and Heard 2009). Together, there is a general relationship between changes that take place on the $\mathrm{Xi}$, but some of these events may occur in a different order at different locations on the chromosome and the temporal order of these events with respect to the switch to late replication is difficult to define precisely. Nonetheless, controlled X-inactivation using an Xist transgene has defined a transition from a reversible initiation step to committed irreversible $\mathrm{X}$-inactivation that coincides with the replication timing switch (Keohane et al. 1996; Wutz and Jaenisch 2000). Hence, it is difficult to formulate a precise hypothesis regarding causal relationships of replication timing to other chromatin changes, but late replication appears to be somehow related to the stability of the silenced state.

\section{Developmental regulation on autosomes}

Compared to the regulation of the $\mathrm{X}$ chromosome, relatively less is known about regulation of autosomes during development. Laborious work by many investigators identified a few dozen loci that replicate at 
different times in different cell lines (Gilbert 2002). From these studies, a general picture emerged that when a tissue-specific gene locus is subject to replication timing regulation, the locus is almost always early replicating in cell types in which it is expressed, whereas it is late replicating when transcriptionally silent. However, these studies also found many gene loci that remained early replicating in all cell types. Furthermore, most were inferred from the comparison of stable, transformed cell lines that represent different tissues, which may have acquired properties during long-term culture that are not seen in the tissues of origin. The key to solving these uncertainties was the advent of homogeneous differentiation systems that could elicit timing changes in response to media conditions (Hiratani et al. 2004; Perry et al. 2004). Moreover, genome-wide microarray technology and its application to replication studies (MacAlpine and Bell 2005) allowed for the possibility to generate a complete description of autosomal replication timing changes. In fact, recent genome-wide studies directly demonstrated that autosomal replication timing changes are widespread during mammalian development. Neural differentiation of mouse ESCs is accompanied by replication timing changes affecting $\sim 20 \%$ of the genome, with smaller differentially replicating domains consolidating into larger coordinately replicated units (Hiratani et al. 2008). A similar fraction of the genome was found to differ in replication timing between embryonic versus wing disk cell lines in Drosophila (Schwaiger et al. 2009). Intriguingly, changes in replication timing were coordinated with transcription changes at the level of large chromosomal domains and rearrangements in sub-nuclear positioning (Hiratani et al. 2008; Williams et al. 2006). The relationship during differentiation demonstrates that changes in replication timing, transcription and subnuclear positioning are dynamically associated, and not related merely because they are correlates of static properties of the genome. Using a relatively synchronous differentiation system, the order of events could be addressed, revealing that the order of transcription vs. replication timing changes is locus-specific, whereas sub-nuclear position changes occurred only when replication timing changes traverse the mid-late stages of S-phase (Hiratani et al. 2010). Hence, there is a general coordination of events, but no specific kinetic relationship. Nonetheless, the fact that these associated properties change coordinately suggests a novel form of genome reorganization in the nucleus during differentiation.

These studies implied that genome-wide replication timing profiles are cell-type specific and consequently that $20 \%$ is almost certainly an underestimate of chromosomal regions subject to replication timing regulation during development. Consistently, recent genome-wide analyses of cell culture systems that model early mouse development indicate that replication timing regulation collectively affects at least $45 \%$ of the genome at some point during differentiation to different germ layers, creating cell-type-specific replication profiles (Hiratani et al. 2010). Interestingly, these experiments revealed that a significant number of early-to-late replication timing changes occurred in a lineage-independent manner and were completed at a stage equivalent to the postimplantation epiblast, which were subsequently stably maintained in downstream lineages (Hiratani et al. 2010). The distinction lies at the epiblast stage, with late postimplantation epiblast resembling downstream germ layer cell types, while early epiblast stage cells more closely resemble pluripotent cells of the inner cell mass (i.e. ESCs). Intriguingly, the replication timing switches in the epiblast occur during a period of little transcriptional change, implying that the replication switches are independent of large-scale changes in transcription. Moreover, lineageindependent, early-to-late replication timing changes coincide with movement of loci toward the nuclear periphery and were difficult to reverse once in place, being stably retained in cells returned to ESC medium and in cell lines that failed to fully revert to the induced pluripotent stem cell (iPSC) state in reprogramming experiments (i.e. cells known as partially reprogrammed iPSCs). In contrast, fully reprogrammed iPSCs reacquire a replication timing program indistinguishable from ESCs (Hiratani et al. 2008, 2010). These results suggest that lineage-independent autosomal replication timing switches occur at the same time and are as developmentally stable as late replication of the $\mathrm{Xi}$. The similar time windows of events imply that the mechanisms regulating lineageindependent replication timing switches on the $\mathrm{Xi}$ and autosomes may cross-talk in the epiblast. However, additional lineage-specific replication timing changes continued to occur in more committed cell types, implying mechanisms exist that function throughout development. 


\section{Reconciling developmental regulation of replication timing with its relationship to static features of chromosomes}

Studies of replication timing have identified a correlation to static features of chromosomes such as isochore GC content and chromosome banding patterns (Bickmore and Craig 1997; Craig and Bickmore 1993; Holmquist et al. 1982; Latt 1977). In fact, one report claimed a "perfect" match of replication timing to isochore GC content (Schmegner et al. 2007). Indeed, an attempt to reconcile static properties with the dynamic changes that occur during development is befuddling. However, in the case of isochore GC content, recent genome-wide analyses have resolved this dilemma by demonstrating that the relationship is far from perfect and is in fact quite cell-type specific (Hiratani et al. 2008, 2010). Moreover, the correlation is considerably stronger in mouse vs. human cells of the same type (T. Ryba, I.H. and DMG, unpublished).

In the case of $R$ and $G$ banding patterns, the question arises as to just how static they are across different tissues. Although the primary sequence of chromosomes has been shown to be a large determinant of banding patterns (Bobrow and Madan 1973; Korenberg and Engels 1978), other factors of chromosome structure must contribute as distinctive patterns can be seen between genetically identical active and inactive $\mathrm{X}$ chromosomes. However, to date, the only evidence that reverse/Giemsa $(R / G)$ bands can change to coincide with developmental replication timing changes is during the inactivation of the $\mathrm{X}$ chromosome (Baranovskaya et al. 1972; Kanda 1973; Sarto et al. 1974). The most logical explanation for the apparent static coincidence of replication timing with $\mathrm{R} / \mathrm{G}$ bands is that differences in replication timing encompass regions that are too small to detect by cytogenetic analyses. In fact, high-resolution banding methods have still not been applied to a systematic comparison of different tissues. Even those techniques with the highest resolution produce average band widths of $1.5 \mathrm{Mb}$ (Yunis 1981), which still may not be sufficient to resolve replication timing switching domains $(400-800 \mathrm{~Kb})$. It is likely that a greater number of bands exist than have been resolved with existing cytogenetic methods, since bands are scored using a threshold staining intensity whereas a range of intensity exists within bands (Bickmore and Craig 1997). In fact, segments of bands with different intensities have been shown to replicate at different times (Ganner and Evans 1971). Hence, the question remains open as to whether or not cytogenetic methods of a resolution sufficient to detect replication domains would reveal tissue-specific chromosome banding patterns. Moreover, a simple inspection of the now-available genome-wide replication profiles (http://www.replicationdomain.org; Weddington et al. 2008) reveals a rather poor correspondence of replication timing to $R$ and $G$ bands. What will be necessary to resolve these issues will be a direct comparison of high-resolution banding patterns to replication timing profiles in the same cell line.

\section{How do developmental cues impinge upon replication timing regulation?}

Ultimately, developmental cues must impinge upon replication timing regulation to induce the changes observed during differentiation. Elucidation of this process, however, requires an understanding of the regulation of replication timing, which remains an enigma. Moreover, at any given transition during differentiation, replication timing switches involve a specific group of replication domains, hence the regulation is most likely mediated through changes in the chromatin substrate of the affected domains rather than changes in global cell cycle pathways such as cyclin-dependent kinases or checkpoint control (Katsuno et al. 2009). Nonetheless, insights from multiple species suggest that regulation influences replication origin firing at a domain-wide level, rather than individual origins controlling the replication timing of each domain. Unraveling how developmental cues influence domain-wide properties of chromosomes will ultimately lead to a deeper understanding of not only replication timing, but also sub-nuclear position and higher-order chromosome structure and how the interactions of these properties regulate transcriptional competence.

1. The timing decision point (TDP) offers an opportunity for altering the replication program

One potential target of a developmentally driven toggle to modulate replication timing would be the timing decision point. After pre-replication complexes are loaded onto chromatin during telophase, 
the timing program for the subsequent S-phase is established at the TDP, typically $1-3 \mathrm{~h}$ into G1phase in mammals (Dimitrova and Gilbert 1999) and at some point between mitosis and START in budding yeast (Raghuraman et al. 1997). The TDP was originally demonstrated in mammals by experiments introducing cell nuclei into Xenopus egg extracts. Nuclei isolated early in G1 could not maintain the normal program while those isolated later in G1 consistently maintained the regular temporal pattern of replication (Dimitrova and Gilbert 1999). The concept of the TDP implies that timing is re-established in each cell cycle and hence this cell cycle regulation may offer a point of intervention for developmentally regulated changes in replication timing.

At present, the mechanisms regulating timing at the TDP are unknown. However, having a specified narrow temporal window during the cell cycle has allowed for the identification of specific components that, being already in place prior to the TDP, are not sufficient to dictate the timing program. For example, pre-replication complex formation, the presence of several histone modifications and the resumption of transcription are all upstream of the TDP (Dimitrova and Gilbert 1999; Wu et al. 2006). The most obvious event that takes place coincident with the TDP is the anchorage of chromosomal domains at specific sub-nuclear locations (Dimitrova and Gilbert 1999). In addition, the mobility of chromatin is relatively high only during early G1-phase, while chromatin motion is locally constrained during the rest of the interphase (Thomson et al. 2004; Walter et al. 2003). Limiting mobility of chromosomal domains could allow for the compartmentalization of enzymes that influence domain-wide chromatin structure, consequently determining the accessibility of resident replication origins to initiation factors (Gilbert 2001; Hayashi et al. 2009). Developmental cues, therefore, may elicit domain-wide changes in chromosome structure by repositioning those domains at the TDP. Consistent with this model, experimentally induced changes in gene position mediated by the inducible targeting of a genetic locus to the nuclear envelope required passage through mitosis and took place during early G1-phase (Kumaran and Spector 2008). This model predicts that repositioning of domains would occur at the TDP prior to a timing switch. Synchronous differentiation systems such as the one discussed above should eventually allow this to be tested.

2. Regulation is at the level of large chromosomal domains

While it may seem logical for replication timing changes to be exerted locally at the level of individual replication origins, either by activating or repressing a tissue-specific origin or by modifying the firing time of an active origin, this concept is difficult to reconcile with our current understanding of metazoan replication origins. First, replication origin selection is independent of replication timing regulation. Using the same Xenopus egg extract system described above, selection of origins was shown to take place at a G1 time point distinctively after the TDP (at the origin decision point; Dimitrova and Gilbert 1999; Dimitrova et al. 2002; Li et al. 2003; Wu and Gilbert 1996). In addition, developmental regulation of replication timing at the beta-globin locus is conserved between human and mouse, but origin site usage is very different; in human cells initiation is confined to one of two closely spaced origins (Aladjem et al. 1998; Kitsberg et al. 1993) while replication initiates from a dispersed set of origins in mice (Aladjem et al. 2002). Second, positions of initiation sites are quite flexible even among cells within a population of the same type (Aladjem 2007; Norio et al. 2005; Takebayashi et al. 2001). Hence, different cells use a different cohort of origins in each cell cycle, so that any mechanism would have to control timing regardless of individual cell origin choice. Taken together, a single-replicon level regulation of replication timing is difficult to reconcile with the contemporary view of metazoan replication origins. However, because our knowledge base on replication origins is still inadequate, regulation at the single-origin level cannot be totally excluded, particularly as an isolated mechanism in specific regions. In fact, a single very strong artificial initiation site was capable of eliciting a modest change in replication timing (Goren et al. 2008).

The available evidence is more consistent with a domain-level regulation of timing and localized regulation of origin efficiency within a domain. During neural differentiation of both human and mouse ESCs, changes in replication timing typically occurred at the level of 400-800 kb domains (Hiratani et al. 2008, 2010; T. Ryba and D.M.G., unpublished). 
Given that a single replicon will duplicate 100 $200 \mathrm{~kb}$ within $1 \mathrm{~h}$, this necessitates the coordinated temporal regulation of at least two or three adjacent origins for most of these switches. This implies an existence of some form of a domain-level regulation, rather than a localized replication timing regulation at the level of a single origin. Interestingly, a recent study in fission yeast showed that heterochromatic pericentromeres and the mat locus were early replicating (Kim et al. 2003) in a Swi6-dependent manner (Hayashi et al. 2009). Swi6 is a heterochromatin protein 1 (HP1) ortholog that is enriched in these heterochromatic domains and regulates replication timing through the loading of the replication initiation factor, Sld3 (Hayashi et al. 2009). Although these fission yeast heterochromatin loci are unusual in that they replicate early, it is possible that the replication timing of mammalian chromosomal domains is regulated by initiation factor recruitment via domainwide factors like HP1. In Drosophila, an HP1 knockdown advances replication timing of centromeric heterochromatin, whereas it delays replication timing of pericentric repeats and chromosome 4, all of which are normally bound by HP1 (M. Schwaiger and D. Schübeler, personal communication). It should be noted that the specific case of HP1 and Sld3 does not necessarily apply to the mammalian system; no mammalian Sld3 orthologs have been reported, and HP1 does not regulate the late replication timing of pericentric heterochromatin in mice (Wu et al. 2006). However, there are many candidates for proteins that are enriched in a domain-wide fashion. For example, many indirect observations suggest that a competition between histone $\mathrm{H} 1 \mathrm{vs}$. high mobility group proteins I and $\mathrm{Y}$ (HMG-I/Y) proteins across large chromosomal segments may regulate replication timing during differentiation (Flickinger 2001).

\section{Cis regulation of domains and genomic context}

Studies in budding yeast clearly show that cisacting information exists that regulates the time of activation of origins independent of their initiation activity per se (Ferguson and Fangman 1992; Friedman et al. 1996). Consistently, a 200-bp cis element that enforces late replication of a neighboring origin has been identified in fission yeast (Yompakdee and Huberman 2004). However, the mechanism by which $c i s$-acting sequences influence replication time is still unknown. In mammals, a deletion in the vicinity of the locus control region (LCR) of the human beta-globin gene locus has been shown to disrupt the domain's variable replication time in different cell types (Cimbora et al. 2000; Goren and Cedar 2003; Simon et al. 2001). It is thus reasonable to expect that cis-acting regulatory elements for replication timing exist in mammals. However, it has not been easy to pinpoint these sequences. For instance, sequences from within the beta-globin LCR itself that appear to be necessary and sufficient to confer replication timing regulation in the context of a transgene in mice are not necessary for timing in the native chromosomal context (Cimbora et al. 2000; Simon et al. 2001). Thus, cis regulation is dependent on the genomic context.

In agreement with the importance of genomic contexts, chromosomal domains that changed replication timing were AT-rich and above a certain threshold of long interspersed nuclear element 1 (LINE-1) transposon density (Hiratani et al. 2008), confirming an earlier study at the genome-wide scale (Hiratani et al. 2004). Our recent ongoing work provides a refined view (Hitarani et al. 2010): constitutively, early-replicating sequences (i.e. early in all cell types tested) were extremely GC rich, gene rich, and LINE-1 poor, whereas constitutively latereplicating sequences were extremely GC poor, gene poor, and LINE-1 rich. It appears that chromosomal domains with sequence properties intermediate between these two extremes are those subject to replication timing regulation during development. Potentially, subtle changes in the dosage of chromatin proteins that bind rather non-specifically to AT vs. GC-rich regions (as, for example, HMG-I/Y and H1 discussed above) could have a profound influence on the enrichment of those proteins across domains with intermediate GC content, particularly if these proteins are capable of spreading through positive feedback loops that seed multi-protein chromatin structures. Changes in protein dosage could be induced globally in the cell or locally by movement of a domain into a spatial compartment where such proteins are enriched or sequestered. In either case, a rapid domain-wide change in chromatin composition could be seeded by subtle changes in the binding of one or a few proteins. With genome-wide replication timing information in hand, chromosome engineering methods can now be employed to make targeted genetic deletions or insertions that address the interaction between cis- 
acting sequences, genomic environments, and subnuclear compartments.

\section{Roles of replication timing regulation}

The precise role of a replication timing program, and why this program is developmentally regulated remains to be elucidated. However, temporal regulation of genome duplication and the existence of multireplicon domains are conserved from humans to budding and fission yeasts (Hayashi et al. 2007; McCune et al. 2008; Versini et al. 2003). DNA replication is centrally linked to many basic cellular processes that are regulated during the cell cycle and development, and defects in replication timing have been observed in various disease models [reviewed in (Hiratani and Gilbert 2009)]. Recent studies allow us to conclude that widespread developmental replication timing switches occur in flies (Schwaiger et al. 2009), mice (Hiratani et al. 2008), and humans (T. Ryba and D.M.G., unpublished). Moreover, there is significant conservation of the replication timing program when regions of conserved synteny from similar cell types are compared between human and mouse (T. Ryba and D.M.G., unpublished). This conservation is greater than the conservation of relative GC content between these same regions. Whatever its role, whether causal or reflective, replication timing is related to properties held in common across large chromosomal segments, and reflects developmental changes to these properties.

Since chromatin is assembled at the replication fork, the most appealing role for replication timing in regulating gene expression during development is to seed the assembly of different types of chromatin at different times during S-phase. In fact, reporter plasmids injected into early or late S-phase mammalian nuclei were assembled into hyper- or hypoacetylated chromatin, respectively (Zhang et al. 2002). This result demonstrates that the environment within nuclei is significantly different at different times during S-phase. A recent follow-up study strengthens this scenario. Taking advantage of the fact that bovine papilloma virus plasmids replicate at different times in consecutive cell cycles (Gilbert and Cohen 1987), it was shown that hypoacetylated latereplicating chromatin becomes hyperacetylated when the mini-circle is replicated early in the subsequent cell cycle (Lande-Diner et al. 2009). Their results suggested that preacetylated histone H4 is actively deacetylated only during late S-phase, while unmodified histone $\mathrm{H} 3$ is actively acetylated by an unknown histone acetylase during early S. Interestingly, acetylation of H4K16, which is enriched in earlyreplicating DNA but depleted in late-replicating regions (Schwaiger et al. 2009), has been shown to prevent chromatin compaction (Corona et al. 2002; Robinson et al. 2008; Shogren-Knaak et al. 2006; Suganuma et al. 2008). Thus, while evidence is still limited, this longstanding hypothesis remains the most likely model that can place timing control as an upstream regulator of chromatin structure and explain the correlation observed in these two properties. In addition, such a system would be ideal for regulating developmental changes, allowing the local modifications to cis elements made at the TDP to spread broad high-order structural changes to domains on the order of several hundred kilobases in a single cell cycle.

Acknowledgment Research in the laboratory of D.M.G. is supported by the NIH grant GM083337. We thank B. Chadwick for critical reading of the manuscript.

\section{References}

Aladjem MI (2007) Replication in context: dynamic regulation of DNA replication patterns in metazoans. Nat Rev Genet 8:588-600

Aladjem MI, Rodewald LW, Kolman JL, Wahl GM (1998) Genetic dissection of a mammalian replicator in the human beta-globin locus [see comments]. Science 281:1005-1009

Aladjem MI, Rodewald LW, Lin CM et al (2002) Replication initiation patterns in the beta-globin loci of totipotent and differentiated murine cells: evidence for multiple initiation regions. Mol Cell Biol 22:442-452

Baranovskaya LT, Zakharov AF, Dutrillaux B et al (1972) Differentiation of $\mathrm{X}$ chromosomes by despiralization methods using 5-bromodeoxyuridine (BUDR) and controlled thermal denaturation. Ann Genet 15:271-274

Barr ML, Bertram EG (1949) A morphological distinction between neurones of the male and female, and the behaviour of the nucleolar satellite during accelerated nucleoprotein synthesis. Nature 163:676

Berezney R, Dubey DD, Huberman JA (2000) Heterogeneity of eukaryotic replicons, replicon clusters, and replication foci. Chromosoma 108:471-484

Bickmore WA, Craig J (1997) Chromosome bands: patterns in the genome. Landes Bioscience, Georgetown

Bobrow M, Madan K (1973) The effects of various banding procedures on human chromosomes, studied with acridine orange. Cytogenet Cell Genet 12:143-156 
Campbell KH, McWhir J, Ritchie WA, Wilmut I (1996) Sheep cloned by nuclear transfer from a cultured cell line. Nature 380:64-66

Chadwick BP, Willard HF (2003) Barring gene expression after XIST: maintaining facultative heterochromatin on the inactive X. Semin Cell Dev Biol 14:359-367

Chadwick BP, Willard HF (2004) Multiple spatially distinct types of facultative heterochromatin on the human inactive $\mathrm{X}$ chromosome. Proc Natl Acad Sci U S A 101:17450-17455

Chow J, Heard E (2009) X inactivation and the complexities of silencing a sex chromosome. Curr Opin Cell Biol 21:359-366

Cimbora DM, Schubeler D, Reik A et al (2000) Long-distance control of origin choice and replication timing in the human beta-globin locus are independent of the locus control region. Mol Cell Biol 20:5581-5591

Corona DF, Clapier CR, Becker PB, Tamkun JW (2002) Modulation of ISWI function by site-specific histone acetylation. EMBO Rep 3:242-247

Craig JM, Bickmore WA (1993) Chromosome bands-flavours to savour. BioEssays 15:349-354

Desprat R, Thierry-Mieg D, Lailler N et al (2009) Predictable dynamic program of timing of DNA replication in human cells. Genome Res; in press

Dimitrova DS, Gilbert DM (1999) The spatial position and replication timing of chromosomal domains are both established in early G1-phase. Mol Cell 4:983-993

Dimitrova DS, Prokhorova TA, Blow JJ, Todorov IT, Gilbert DM (2002) Mammalian nuclei become licensed for DNA replication during late telophase. J Cell Sci 115:51-59

Farkash-Amar S, Lipson D, Polten A et al (2008) Global organization of replication time zones of the mouse genome. Genome Res 18:1562-1570

Ferguson BM, Fangman WL (1992) A position effect on the time of replication origin activation in yeast. Cell 68:333-339

Flickinger R (2001) Replication timing and cell differentiation. Differentiation 69:18-26

Fraser P, Bickmore W (2007) Nuclear organization of the genome and the potential for gene regulation. Nature 447:413-417

Friedman KL, Diller JD, Ferguson BM et al (1996) Multiple determinants controlling activation of yeast replication origins late in S phase. Genes Dev 10:1595-1607

Ganner E, Evans HJ (1971) The relationship between patterns of DNA replication and of quinacrine fluorescence in the human chromosome complement. Chromosoma 35:326-341

Gierman HJ, Indemans MH, Koster J et al (2007) Domain-wide regulation of gene expression in the human genome. Genome Res 17(9):1286-1295

Gilbert DM (2001) Nuclear position leaves its mark on replication timing. J Cell Biol 152:F11-F16

Gilbert DM (2002) Replication timing and transcriptional control: beyond cause and effect. Curr Opin Cell Biol 14:377-383

Gilbert DM, Cohen SN (1987) Bovine papilloma virus plasmids replicate randomly in mouse fibroblasts throughout S-phase of the cell cycle. Cell 50:59-68

Gilbert DM, Gasser SM (2006) Nuclear structure and DNA replication. In: DePamphilis ML (ed) DNA replication and human disease. Cold Spring Harbor Laboratory, Cold Spring Harbor

Goren A, Cedar H (2003) Replicating by the clock. Nat Rev Mol Cell Biol 4:25-32
Goren A, Tabib A, Hecht M, Cedar H (2008) DNA replication timing of the human beta-globin domain is controlled by histone modification at the origin. Genes Dev 22:13191324

Grimm OH, Gurdon JB (2002) Nuclear exclusion of Smad2 is a mechanism leading to loss of competence. Nat Cell Biol $4: 519-522$

Gurdon JB (2006) From nuclear transfer to nuclear reprogramming: the reversal of cell differentiation. Annu Rev Cell Dev Biol 22:1-22

Hayashi M, Katou Y, Itoh T et al (2007) Genome-wide localization of pre-RC sites and identification of replication origins in fission yeast. EMBO J 26:1327-1339

Hayashi MT, Takahashi TS, Nakagawa T, Nakayama J, Masukata H (2009) The heterochromatin protein Swi6/HP1 activates replication origins at the pericentromeric region and silent mating-type locus. Nat Cell Biol 11:357-362

Hiratani I, Gilbert DM (2009) Replication timing as an epigenetic mark. Epigenetics 4:93-97

Hiratani I, Leskovar A, Gilbert DM (2004) Differentiationinduced replication-timing changes are restricted to ATrich/long interspersed nuclear element (LINE)-rich isochores. Proc Natl Acad Sci U S A 101:16861-16866

Hiratani I, Ryba T, Itoh M et al (2008) Global reorganization of replication domains during embryonic stem cell differentiation. PLoS Biol 6:e245

Hiratani I, Takebayashi S, Lu J, Gilbert DM (2009) Replication timing and transcriptional control: beyond cause and effect-part II. Curr Opin Genet Dev 19:142-149

Hiratani I, Ryba T, Itoh M et al (2010) Genome-wide dynamics of replication timing revealed by in vitro models of mouse embryogenesis. Genome Res; in press

Hochedlinger K, Plath K (2009) Epigenetic reprogramming and induced pluripotency. Development 136:509-523

Holmquist G, Gray M, Porter T, Jordan J (1982) Characterization of Giemsa dark- and light-band DNA. Cell 31:121129

Kanda N (1973) A new differential technique for staining the heteropycnotic X-chromosome in female mice. Exp Cell Res 80:463-467

Karnani N, Taylor C, Malhotra A, Dutta A (2007) Pan-S replication patterns and chromosomal domains defined by genome-tiling arrays of ENCODE genomic areas. Genome Res 17:865-876

Katsuno Y, Suzuki A, Sugimura K et al (2009) Cyclin A-Cdk1 regulates the origin firing program in mammalian cells. Proc Natl Acad Sci U S A 106:3184-3189

Keohane AM, O'Neill LP, Belyaev ND, Lavender JS, Turner BM (1996) X-Inactivation and histone $\mathrm{H} 4$ acetylation in embryonic stem cells. Dev Biol 180:618-630

Kim SM, Dubey DD, Huberman JA (2003) Early-replicating heterochromatin. Genes Dev 17:330-335

Kitsberg D, Selig S, Keshet J, Cedar H (1993) Replication structure of the human $\beta$-globin gene domain. Nature 368:588-590

Korenberg JR, Engels WR (1978) Base ratio, DNA content, and quinacrine-brightness of human chromosomes. Proc Natl Acad Sci U S A 75:3382-3386

Kumaran RI, Spector DL (2008) A genetic locus targeted to the nuclear periphery in living cells maintains its transcriptional competence. J Cell Biol 180:51-65 
Lande-Diner L, Zhang J, Cedar H (2009) Shifts in replication timing actively affect histone acetylation during nucleosome reassembly. Mol Cell 34:767-774

Latt SA (1977) Fluorescent probes of chromosome structure and replication. Can J Genet Cytol 19:603-623

Li B, Carey M, Workman JL (2007) The role of chromatin during transcription. Cell 128:707-719

Li F, Chen J, Solessio E, Gilbert DM (2003) Spatial distribution and specification of mammalian replication origins during G1 phase. J Cell Biol 161:257-266

Lin H, Gupta V, Vermilyea MD et al (2007) Dosage compensation in the mouse balances up-regulation and silencing of X-linked genes. PLoS Biol 5:e326

MacAlpine DM, Bell SP (2005) A genomic view of eukaryotic DNA replication. Chromosome Res 13:309-326

MacAlpine DM, Rodriguez HK, Bell SP (2004) Coordination of replication and transcription along a Drosophila chromosome. Genes Dev 18:3094-3105

McCune HJ, Danielson LS, Alvino GM et al (2008) The temporal program of chromosome replication: genomewide replication in clb5 \{Delta\} Saccharomyces cerevisiae. Genetics 180:1833-1847

Meshorer E, Misteli T (2006) Chromatin in pluripotent embryonic stem cells and differentiation. Nat Rev Mol Cell Biol 7:540-546

Morishima A, Grumbach MM, Taylor JH (1962) Asynchronous duplication of human chromosomes and the origin of sex chromatin. Proc Natl Acad Sci U S A 48:756-763

Norio P, Kosiyatrakul S, Yang Q et al (2005) Progressive activation of DNA replication initiation in large domains of the immunoglobulin heavy chain locus during B cell development. Mol Cell 20:575-587

Perry P, Sauer S, Billon N et al (2004) A dynamic switch in the replication timing of key regulator genes in embryonic stem cells upon neural induction. Cell Cycle 3:1645-1650

Raghuraman M, Brewer B, Fangman W (1997) Cell cycledependent establishment of a late replication program. Science 276:806-809

Robinson PJ, An W, Routh A et al (2008) $30 \mathrm{~nm}$ chromatin fibre decompaction requires both $\mathrm{H} 4-\mathrm{K} 16$ acetylation and linker histone eviction. J Mol Biol 381:816-825

Sarto GE, Therman E, Patau K (1974) Increased Q fluorescence of an inactive Xq-chromosome in man. Clin Genet 6:289293

Schmegner C, Hameister H, Vogel W, Assum G (2007) Isochores and replication time zones: a perfect match. Cytogenet Genome Res 116:167-172

Schubeler D, Scalzo D, Kooperberg C et al (2002) Genome-wide DNA replication profile for Drosophila melanogaster: a link between transcription and replication timing. Nat Genet $32: 438-442$

Schwaiger M, Stadler MB, Bell O et al (2009) Chromatin state marks cell-type- and gender-specific replication of the Drosophila genome. Genes Dev 23:589-601

Shogren-Knaak M, Ishii H, Sun JM et al (2006) Histone H4K16 acetylation controls chromatin structure and protein interactions. Science 311:844-847

Simon I, Tenzen T, Mostoslavsky R et al (2001) Developmental regulation of DNA replication timing at the human beta globin locus. Embo J 20:6150-6157
Snow MHL (1977) Gastrulation in the mouse: growth and regionalization of the epiblast. J Embryol Exp Morphol 42:293-303

Steinbach OC, Wolffe AP, Rupp RA (1997) Somatic linker histones cause loss of mesodermal competence in Xenopus. Nature 389:395-399

Suganuma T, Gutierrez JL, Li B et al (2008) ATAC is a double histone acetyltransferase complex that stimulates nucleosome sliding. Nat Struct Mol Biol 15:364-372

Takagi N, Sugawara O, Sasaki M (1982) Regional and temporal changes in the pattern of $\mathrm{X}$-chromosome replication during the early post-implantation development of the female mouse. Chromosoma 85:275-286

Takahashi K, Yamanaka S (2006) Induction of pluripotent stem cells from mouse embryonic and adult fibroblast cultures by defined factors. Cell 126:663-676

Takebayashi SI, Manders EM, Kimura H, Taguchi H, Okumura K (2001) Mapping sites where replication initiates in mammalian cells using DNA fibers. Exp Cell Res 271:263-268

Thomson I, Gilchrist S, Bickmore WA, Chubb JR (2004) The radial positioning of chromatin is not inherited through mitosis but is established de novo in early G1. Curr Biol $14: 166-172$

Versini G, Comet I, Wu M et al (2003) The yeast Sgs1 helicase is differentially required for genomic and ribosomal DNA replication. Embo J 22:1939-1949

Waddington $\mathrm{CH}$ (1940) Organisers and genes. Cambridge University, Cambridge

Walter J, Schermelleh L, Cremer M, Tashiro S, Cremer T (2003) Chromosome order in HeLa cells changes during mitosis and early G1, but is stably maintained during subsequent interphase stages. J Cell Biol 160:685-697

Weddington N, Stuy A, Hiratani I et al (2008) ReplicationDomain: a visualization tool and comparative database for genomewide replication timing data. BMC Bioinformatics 9:530

White EJ, Emanuelsson O, Scalzo D et al (2004) DNA replication-timing analysis of human chromosome 22 at high resolution and different developmental states. Proc Natl Acad Sci U S A 101:17771-17776

Williams RR, Azuara V, Perry P et al (2006) Neural induction promotes large-scale chromatin reorganisation of the Mash1 locus. J Cell Sci 119:132-140

Woodfine K, Fiegler H, Beare DM et al (2004) Replication timing of the human genome. Hum Mol Genet 13:191-202

Wu J-R, Gilbert DM (1996) A distinct G1 step required to specify the chinese hamster DHFR replication origin. Science 271:1270-1272

Wu R, Singh PB, Gilbert DM (2006) Uncoupling global and fine-tuning replication timing determinants for mouse pericentric heterochromatin. J Cell Biol 174:185-194

Wutz A, Jaenisch R (2000) A shift from reversible to irreversible $\mathrm{X}$ inactivation is triggered during ES cell differentiation. Mol Cell 5:695-705

Yompakdee C, Huberman JA (2004) Enforcement of late replication origin firing by clusters of short G-rich DNA sequences. J Biol Chem 279:42337-42344

Yunis JJ (1981) Mid-prophase human chromosomes. The attainment of 2000 bands. Hum Genet 56:293-298

Zhang J, Xu F, Hashimshony T, Keshet I, Cedar H (2002) Establishment of transcriptional competence in early and late S phase. Nature 420:198-202 